\title{
Ultraschallsensoren für die Instandhaltung
}

\author{
Peter Holstein ${ }^{1}$, Nicki Bader ${ }^{1}$, Christian Probst ${ }^{1}$, Steffen Seitz ${ }^{2}$ \\ 1 SONOTEC GmbH, Nauendorfer Str. 2, 06102 Halle \\ ${ }^{2} T U$ Dresden, Mommsenstr. 1201062 Dresden
}

\section{Zusammenfassung}

Im Beitrag wird ein Konzept und dessen Realisierung vorgestellt, dass die Erfassung von Ultraschall in einem breiten Frequenzbereich ermöglicht. Die physikalischen Grundlagen der akustischen Vorgänge im Ultraschallbereich wurden analysiert, um darauf aufbauend ein neues Sensor- und Messgerätekonzept zu entwickeln und technologisch umzusetzen. Sensorische Grundlage hierfür sind neu entwickelte Sensoren, die für einen breiteren Frequenzbereich einsetzbar sind. Damit sind Vorgänge neben den bisher hauptsächlich bewerteten zeitlichen Fluktuationen auch spektral bewertbar. Dies erweitert die Möglichkeiten für den Einsatz komplexerer Datenbewertungen erheblich. Die Körperschallsensoren basieren dabei auf sogenannten piezoelektrischen Kompositmaterialien. Der Frequenzgang dieser Sensoren ermöglicht die spektrale Nutzung der aufgezeichneten Signale. Damit steht eine breite Palette an Signalverarbeitungsoptionen zur Verfügung, die sowohl in Echtzeit als auch für aufwendigere Postprocessing-Verarbeitung genutzt werden können. Die Vorteile der Sensorik kommen auch dadurch zum Tragen, dass viele der gegenwärtig verfügbaren modernen Technologien integriert werden. Dies betrifft Elektronik, embedded IT, Software zur Visualisierung und Kommunikation und industriell orientierte Mathematik aber auch Design, Ergonomie und Produzierbarkeit. Auf Basis dieser Sensoren wurde deshalb eine neue Plattform für Prüftechnik für die Instandhaltung konzipiert, die neue Features und Möglichkeiten bietet- insbesondere im Hinblick auf die Kundenspezifik von Lösungen.

Im Beitrag wird der Nutzen des neuen Sensorkonzepts für die Erschließung neuer Anwendungen vorgestellt. Insbesondere werden die Daten genutzt, um mit Verfahren des maschinellen Lernens bessere Voraussagen zu Lebensdauern, zur Bewertung von Betriebszuständen und Maschinenteilen zu generieren.

Keywords: Ultraschall, Körperschallsensor, Instandhaltung, maschinelles Lernen

\section{Motivation}

Ultraschall spielt im Kontext der Methoden der Technischen Akustik in der Instandhaltung eine sehr spezialisierte Rolle. Dies widerspiegelt sich darin, dass nur relativ einfache Verfahren auf wenige ausgewählte Anwendungsfälle ausgerichtet sind. Beispiele sind die Suche von Druckluftlecks oder einfache Bewertungen von Fehlern in Kugellagern. Das Finden weiterer Anwendungen wird dabei vor allem durch sehr konservative technologische Lösungsansätze eingeschränkt, die im wesentlichen darauf beruhen, dass schmalbandige ResonanzSensoren eingesetzt werden. Dies sichert zwar eine hohe Sensitivität, schränkt aber andererseits durch die Bandpassbegrenzung die Bewertung komplexer physikalischtechnischer Prozesse erheblich ein. Dies ist symbolisch in Abbildung 1 skizziert. Die rote Linie steht für die Verwendung der Ultraschallverfahren in einem, engen Frequenzbereich (analoge Heterodyn- technologie). Damit ist nur eine sehr begrenzte Einsicht in die technologischen in die physikalischen und technischen Prozesse möglich. Die Grenzen zwischen Verfahren sind oft willkürlich. Der Balken zwischen 10 und 100 $\mathrm{kHz}$ steht für die im Beitrag beschrieben Technik.

Die Erweiterung des Frequenzbereich scheint folgerichtig, wenn man grundlegende physikalische Phänomene wie:

- Turbulenz (Strömungsakustik)

- Reibung (Rauigkeit von Materialpaarungen)

- Mikrorisse und Rissfortschritt

- Kavitation

- Entladungen

- Impulskontakte (lose Bauteile ...)

- Eigenschwingungen kleiner Strukturen 
betrachtet. Für jedes dieser Phänomene kann die Signalverarbeitung angepasst und optimiert werden, um (im Sinne des Einsatzes in der Instandhaltung) Verschleißbewertungen, Trendanalysen, Einsatzentscheidungen und Prognosen sichere Daten zu liefern.

Es sei darauf hingewiesen, dass der Einsatz breitbandiger Sensoren die zu verarbeitenden und anfallenden Datenmengen erheblich vergrößern. Entsprechende intelligente Verfahren zur Reduktion der Datenmenge möglichst in Echtzeit - wie Mustererkennung, automatische Fehlerklassifizierung u.ä. bieten sich im Kontext der aktuellen Entwicklungen der Behandlung großer Datenmengen an.

\section{Gerätekonzept}

Das Gerätekonzept ist unmittelbar mit der Sensorfamilie gekoppelt. Für Prüfaufgaben im industriellen Umfeld - insbesondere in der Instandhaltung - steht ein portabler Messdatensammler zur Verfügung, der für Anwendungen im Frequenzbereich des für die Instandhaltung relevanten Bereich ausgelegt ist und aufgrund der internen Struktur (Schnittstellen, Prozessor, Speicher, Echtzeitmodul, Betriebssystem) äußerst flexibel an verschiedene Messaufgaben angepasst werden kann [1].

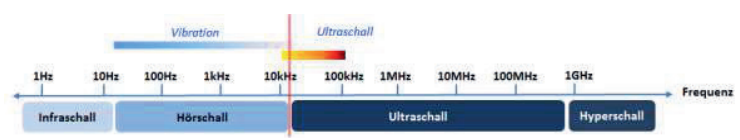

Abb. 1: Einordung der Ultraschallmethoden in der Instandhaltung in das akustische Spektrum.

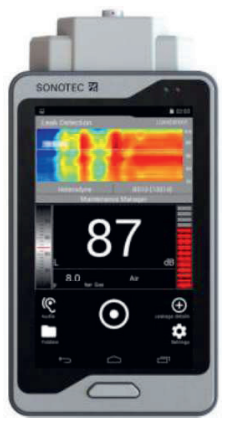

Abb. 2: Basiseinheit für die Ankopplung digitaler Sensoren. Die DAQ-Einheit verfügt über einen integrierten FPGA für Echtzeit.

Die Kombination aus digitaler Prüftechnik und digitaler Sensorik in Verbindung mit der breitbandigen Betrachtung physikalischer Vorgänge ist in der Instandhaltung relativ neu
[2,3]. Entsprechend sind Verfahren, Algorithmen und Normen usw. erst im Entstehen.

\section{Luftschallsensoren}

Luftultraschallanwendungen werden in der Instandhaltung in zwei generellen Betriebsmodi eingesetzt. Zum einen wird direkt von einem technischen System erzeugter Ultraschall nachgewiesen und bewertet. Zum anderen kann Ultraschall aktiv von einem Ultraschallsender abgestrahlt und dann entsprechend der Prüfaufgabe in einiger Distanz nachgewiesen werden. Eine typische Anwendung ist die Prüfung technischer Systeme auf Dichtheit. Die Gründe für die Verwendung der Ultraschallfrequenzen sind unterschiedlich. Oft ist in industriellen Umgebungen der Umgebungslärm zu groß um akustische Methoden (zumindest mit einfachen Verfahren) sinnvoll einzusetzen zu können. Ein „Ausweichen" zu höheren Frequenzen kann in wichtigen Fällen Ziel führend sein. Für die Luftschallanwendungen wurde ein ähnliches Konzept für digitale Ultraschallmikrofone entwickelt. Als Wandlerelemente dienen hierbei 1/4" Mikrofonkapseln, die im hochfrequenten Bereich über einen geeigneten Frequenzgang verfügen. In die digitalen Ultraschallmikrofone sind Frequenzfilter, Verstärkungsstufen, A/D-Wandlung und Digitalschnittstelle integriert. Aufgrund der Analogie zu Messmikrophonen sind damit im Rahmen der möglichen Genauigkeit Messungen im Ultraschallbereich möglich, die zu den im Hörschall üblichen Bezugswerten und Berechnungen kompatibel sind. Die Empfindlichkeit dieser digitalen Mikrofone ist mit der von in der Instandhaltung weit verbreiteten einfachen resonanten Empfängern vergleichbar. Allerdings eröffnet der breite Frequenzbereich auch bei Luftultraschall erweiterte Anwendungsmöglichkeiten und bessere Bewertungen [4].

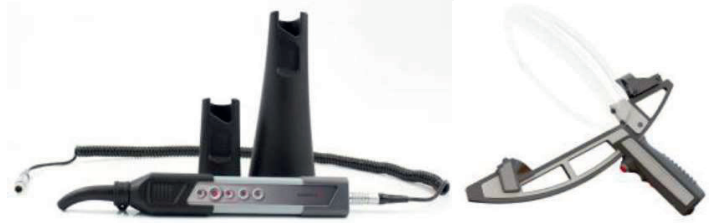

Abb. 3: Links - Digitaler Luftultraschallsensor BS10 mit verschiedenen Aufsätzen zur Suche und Bewertung von Lecks in Druckluftsystemen

Rechts - Kompakte Integration des digitalen Luftultraschallsensors in einen Parabolspiegel zur Messung über größere Distanzen. 


\section{Körperschallsensoren}

Für Anwendungen in der Instandhaltung sind Frequenzen im Bereich von etwa $20 \mathrm{kHz}$ bis $100 \mathrm{kHz}$ von Bedeutung. Damit liegen diese Frequenzen über dem Erfassungsbereich von Schwingungssensoren und unter dem von den für Anwendungen in der akustischen Emission verfügbaren Körperschallsensoren [5].

Ziel der breitbandigen Sensorik ist die Aufwertung der Ultraschalldiagnoseverfahren durch Digitalisierung und Nutzung der spektralen Information. Die Notwendigkeit breitbandiger Körperschallsensorik ergibt sich aus vielen Anforderungen in der Instandhaltung (Maschinendiagnose, Strömungsprobleme).

Kernstück der Körperschallsensoren ist ein Transducerelement aus piezoelektrischem Kompositmaterial. Das Herstellungs- und Funktionsprinzip ist in Abbildung 4 veranschaulicht. Bei monolithischen Transducerelementen tritt eine Vielzahl von Resonanzen auf [2,6], die spektrale Bewertungen erschweren, wohingegen bei der stäbchenförmigen Anordnung der piezoaktiven Elemente in wesentlichen die Dickenschwingung dominiert. Damit ist die Frequenzcharakteristik [2,6] wesentlich "glatter" und Auswertealgorithmen numerisch besser beherrschbar.

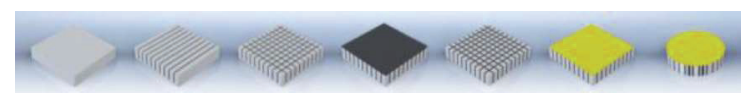

Abb. 4: Technologische Schritte bei der Herstellung des Sensorelements als piezo-elektrische Compositmaterial - Piezokeramikplatte, erster Schnitt, zweiter Schnitt, Einfüllen der Matrix, Schleifen auf Enddicke, Metallisieren und Polarisieren, Zuschnitt der Endgeometrie

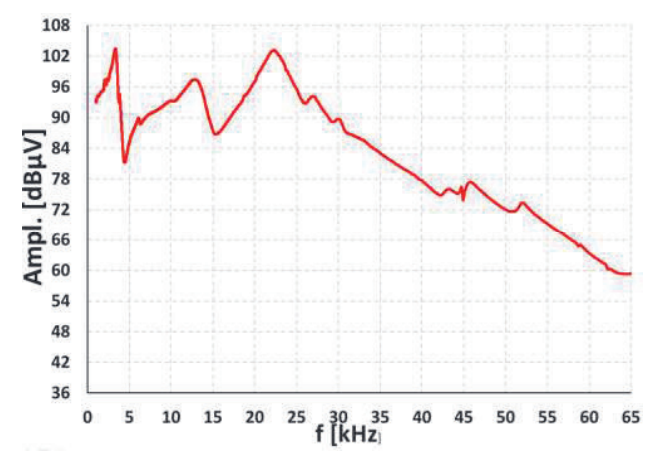

Abb. 5: Frequenzkennlinie des kompletten Sensors T10

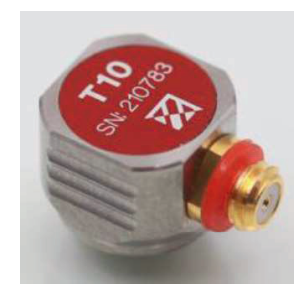

\section{Abb. 6: Ausführungsform des Sensors}

Die digitalen Bauformen der Körperschallsensoren sind für wichtige Anwendungen in der Instandhaltung optimiert. Diverse Waveguides erlauben leichte Kontakte zu Prüflingen und damit schnelle Prüfungen.

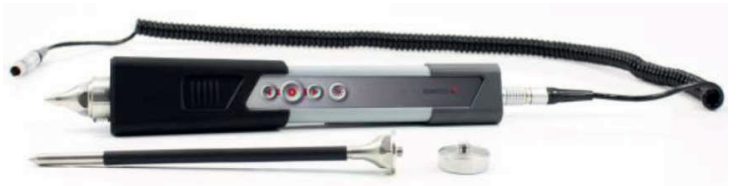

$\begin{array}{llr}\text { Abb. 7: } & \text { Digitale Variante } & \text { des } \\ & \text { Körperschallsensor BS20. Die } \\ \text { unterschiedlichen Kopplungen sind } & \\ \text { an die jeweiligen Anwendungen } \\ \text { angepasst. }\end{array}$

Allerdings ist die Frequenzkennlinie von den Vorläufen beeinflusst. Deshalb werden diese vor allem bei qualitativ orientierten Prüfungen (z. B. an heißen oder schlecht zugänglichen Stellen) eingesetzt.

\section{Kalibrierung der Sensoren}

Die für die Kalibrierung von Schwingungssensoren eingesetzten Systeme sind nicht für Frequenzen ausgelegt, die bis in die niederen Ultraschallfrequenzen reichen. Vibrations- und Ultraschallsensoren unterscheiden sich im Aufbau und der Funktionsweise [5]. Ein wichtiger Unterschied ist die bei den Körperschallsensoren fehlende seismische Masse. Damit ist eine direkte Kalibrierung bezüglich üblicher Bezugsgrößen für Vibrationen schwierig.

Ziel ist es, unter Anwendung und Modifizierung geeigneter Kalibrierverfahren, die Sensoren in einem möglichst großen Frequenzbereich zu kalibrieren und die Reproduzierbarkeit der Kennlinien bei der Herstellung und im Einsatz zu beherrschen. Oft werden die Sensoren mit der Hand für Prüfzwecke gehalten. Dies macht Vergleiche (und Kalibrierungen) besonders herausfordernd. Nach bisherigem Kenntnisstand sind Kalibrierungen bis etwa $60 \mathrm{kHz}$ realisierbar [7]. Kern der Verfahren sind gewisse Optimierungen der Referenzen, die verbunden sind mit entsprechenden 
konstruktiven Anpassungen. Darüber hinaus sind weitere Untersuchungen notwendig. Auf dieser Basis können die Zielparameter für die Modifikation der Kalibrierungsverfahren festgelegt werden.

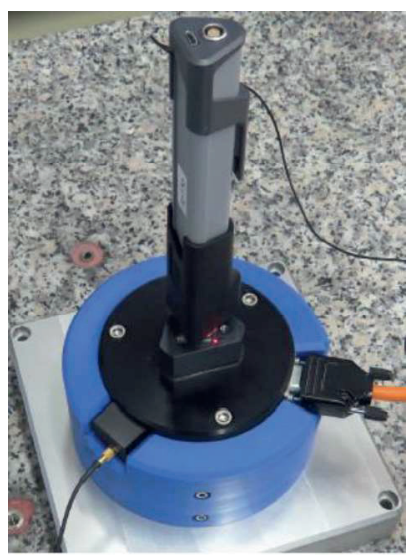

Abb. 8: Schwingregelsystem, hier mit Körperschallsensor BS20 [7]

\section{Anwendungsbeispiel}

Die Körperschallsensoren wurden bei der Instrumentierung von Langzeitversuchen an einen Maschinenprüfstand eingesetzt. Ziel war dabei die Nutzung breitbandiger Ultraschalldaten für die Bewertung entstehender Schäden in Kugellagern. Dafür wurden zum einen Kugellager mit "künstlichen“ Schäden präpariert und zum anderen in Dauerversuchen hoch belastet. Als Input für Lernverfahren wurden die hochfrequent (200 $\mathrm{kHz}$ ) abgetasteten Daten der Körperschallsensoren verwendet. Der Prüfstand wurde mit mehreren Sensoren instrumentiert. Die Belastungsversuche dauerten mehrere Wochen bis zum völligen Versagen der Lager.
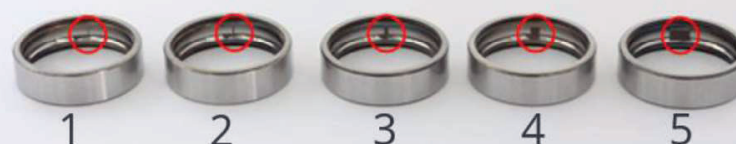

Abb. 9: Präparierte Kugellager; Im Außenring von Kugellagern wurden mit einem Laser definierte Schäden mit wachsendem Ausmaß eingebracht.

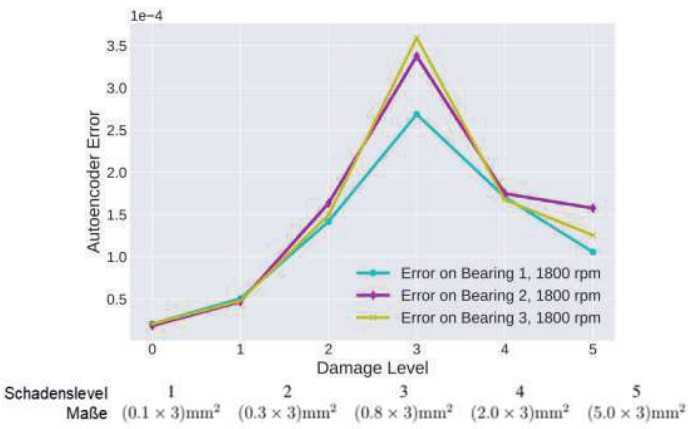

Abb. 10: Darstellung des Autoencoder-Fehlers über dem Schadenslevel

Wird der Schaden ähnlich groß wie die Abmessung der Wälzlager $(4,7 \mathrm{~mm})$ kommt es $\mathrm{zu}$ einer Stoßentlastung, welche vom Autoencoder wieder als geringerer Fehler wahrgenommen wird (siehe Abb. 10) Die Möglichkeit eines solchen Verhaltens wird auch in der VDI3832 [8]. Dies demonstriert, dass vereinfachende Prüfdaten in vielen Fällen nicht zielführend sein werden.

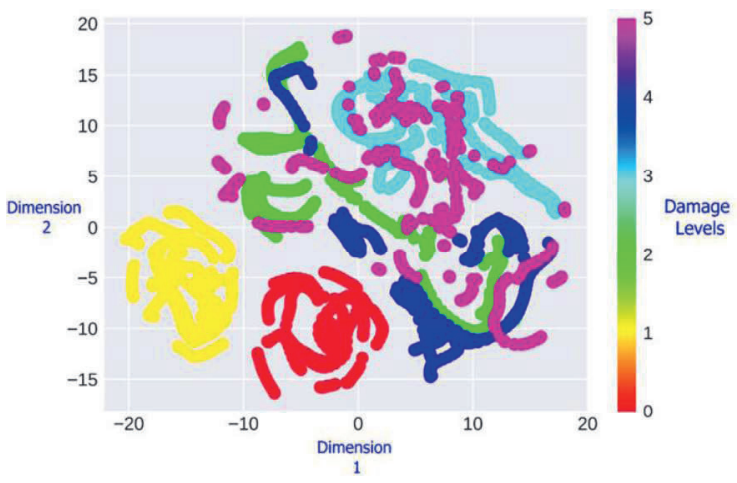

Abb. 11: Plot der im Autoencoder gruppierten Samples der verschiedenen Schadensklassen

Die Gruppierung für Schadensklassen mit niedrigem bzw. keinem Fehler ist deutlich erkennbar (Abb. 11). Damit ist eine Unterscheidung auch von geringen Abweichungen (1) vom unbeschädigten Fall (0) möglich. Die Gruppierung der höheren Schadenszustände ist erkennbar. Die Klassifikation wird aber komplizierter. Es ist allerdings zu erwarten, dass mit der Erhöhung der Datenmenge (Trainigsdaten) die automatischen Auswertungen besser werden [9]. 


\section{Zusammenfassung}

Die Einführung breitbandiger Sensoren für Prüfaufgaben in der Instandhaltung ermöglicht eine deutliche Erweiterung der Anwendungsbreite von Ultraschallverfahren und damit der akustischen Methoden. Deren Anwendung erfordert sowohl für Luftultraschall als auch für Körperschall neue Kalibriermethoden sowie leistungsfähige spezifische Messtechnik und angepasste Algorithmen.

\section{Danksagung}

Die Arbeiten wurden teilweise durch das Bundesministerium für Bildung und Forschung (BMBF-Programm zwanzig20, Förderkennzeichen 03ZZ0518C) und das Bundesministerium für Wirtschaft und Energie (Projekt sUSe - Förderkennzeichen ZF4327503PO8) gefördert.

\section{Literaturnachweis}

[1] https://www.sonotec.de/produkte/vorbeugendeinstandhaltung/produkte/sonaphone/

[2] C. Probst, P. Holstein, Application of ultrasound technology in condition monitoring, Condition Monitor, 372(2018)5-10,

[3] P. Holstein, C. Probst, A. Tharandt, G. Werner, T. Werner, Condition Monitoring with Ultrasound - new Approaches, Proceed. 3rd International Rotating Equipment Conference (IREC), Pumps, Compressors and Vacuum Technology, Düsseldorf, Germany, $14-15$ September 2016, ISBN- 978-3-8163-0697-9

[4] P. Holstein, M. Barth, C. Probst, Acoustic methods for leak detection and tightness testing, Proceedings, 19th World Conference on Non-Destructive Testing; 13 - 17 June 2016 in Munich, Germany

[5] T. Petzsche, Sensing Structural Borne Noise in Solid State Materials and Related Applications, Proceed. 21. Koll. Schallemission, Fulda 2017, https://www.dgzfp.de/Portals/schallemission201 7/BB/20.pdf

L. Soares, M. Dumont, T. Petzsche, Sensing Structural Borne Noise in Solid State Materials and Related Applications, Proceed.

EuroRegio2016, 13.-15.06.2016, Porto,

Portugal http://www.sea-acustica.es/fileadmin/Oporto16/223.pdf

[6] P. Holstein, C. Probst, H. Münch, A. Klepel, A. Tharandt, Application of Acoustic Emission in Machinery and Process Monitoring, Proceed. 31st Conference of the European Working Group on Acoustic Emission (EWGAE), 3.5.9.2014, Dresden, Germany,

[7] P.Holstein, C. Probst, P. Begoff, H. Schulze Kalibrierung von Körperschallsensoren, Fortschritte der Akustik - 44. Deutsche Jahrestagung für Akustik, 19.-22.03. 2018 in München, ISBN-10: 3939296139

[8] VDI 3832, https://www.vdi.de/nc/richtlinie/vdi_3832koerperschallmessungen_zur_zustandsbeurteilung_von_waelzlagern_in _maschinen_und_anlagen/

[9] $\bar{S}$. Seitz, R. Tetzlaff, P. Holstein, C. Probst, Towards Fault Diagnosis with Attention, Proceedings $26^{\text {th }}$ Int. Congr. on Sound and Vibration, 7-11.07.2109, im Druck 